\title{
HOW DO WE EVALUATE AND MANAGE MANY DIFFERENT VACCINATION SCHEDULES IN THE EU?
}

\author{
Vladimír Oleár ${ }^{1,2}$, Zuzana Krištúfková1, Mária Štefkovičová ${ }^{2}$ \\ 'Department of Epidemiology, Faculty of Public Health Studies, Slovak Medical University, Bratislava, Slovak Republic \\ ${ }^{2}$ Faculty of Healthcare, Alexander Dubček University of Trenčín, Trenčin, Slovak Republic
}

\begin{abstract}
SUMMARY
Background: Vaccines are biologic medical products, the biological activity and characteristics of which are significantly different from common drugs and other medical products. The process of determining the dosing (vaccination) schedule for a particular vaccine is based on different principles and rules than other drugs. The dosing schedule for drugs is based on the essential pharmacological properties: pharmacokinetics and pharmacodynamics. When determining the schedule for vaccines, the pharmacokinetic and pharmacodynamic principles cannot be applied: sero-conversion and sero-protectivity of the biologically active component of the vaccine need to be applied. As opposed to drugs and medical products the dosing (vaccination) schedule in the Summary of Product Characteristics (SPC) is often provided in several versions, sometimes with a supplement referring to official (national) recommendations. In relation to the large variability vaccination schedules in the European Union (EU), it is not realistic to test each vaccination schedule in clinical studies. Requiring clinical trials for each vaccination schedule used only for the needs of regulators is more of an ethical issue than a scientific one. The European Centre for Disease Prevention and Control (ECDC), which is the Scientific Panel on Childhood Immunisation Schedule (SPACIS), accepts all the schedules used in EU countries as valid.

Methods and Results: A review of the literature on immunisation schedules for primary series and booster doses choosing the following key words: immunisation, vaccination schedule, primary, booster, timing, vaccination delay.
\end{abstract}

Key words: immunisation, vaccination schedule, primary, booster, vaccination delay

Address for correspondence: V. Oleár, Department of Epidemiology, Faculty of Public Health, Slovak Medical University, Limbová 12, 83303 Bratislava, Slovak Republic. E-mail: vladimirolear@gmail.com

\section{INTRODUCTION}

The principal difference between drugs and vaccines lies in the presentation of the active substance in the organism. Drugs establish a pharmacodynamic and pharmacokinetic profile while vaccines do not with the exception of non-antigen components of vaccines such as excipients (e.g. pharmacokinetics of thiomersal, aluminum, phenol etc.). With respect to vaccines, the pharmacodynamic studies are basically studies of immunogenicity which determine the immune response of the organism to a vaccine (antigen). Pharmacokinetics involves the absorption, distribution, metabolism, and excretion of chemicals administered to organism. Pharmacokinetics often uses mathematical multi-compartment models. Compartments are tissues where the distributed substance achieves specific uniform concentration. Pharmacokinetic compartment is a part of the pharmacokinetic mathematical model and is not anatomically defined (1). Pharmacodynamics is the study of the biochemical and physiological effect of the substance on the organism and the association between the size of the dose and its effect.

Seroconversion is the production of detectable specific antibodies against microorganisms in blood serum, resulting from either infection or immunization. Before infection or administration of antigen to the organism, the antibodies are not detectable in serum or, perhaps some level or concentration of antibodies could be detectable. Usually there is a minimum 4-fold increase of antibody titer compared to the initial levels. After seroconversion, a specific antibody becomes dominant in the serum and the original antigen that caused the seroconversion is no longer detectable in the serum. The generated antigen-antibody immune complexes are rapidly eliminated from the body through several immune mechanisms, so that only the dominant immune complex is detectable in the blood serum. Seroconversion is a binary absolute value; seroprotectivity is the percentage of studied subjects with seroconversion - relative factor. Absence of the antibodies after vaccination does not automatically mean "zero" protection effect. In the case of vaccines, this effect may be mediated by cellular immunity, the level of which is not presented in seroconversion.

Seroprotectivity is the protective effect acquired after immunization or after infection, defined on the basis of the presence of specific antibodies. Seroprotectivity is defined as the percentage of vaccinated persons with the protective titer of antibodies after immunization. Nevertheless, in some vaccines (e.g. pneumococcal vaccine, MMR, BCG), the protective titers are not known. There are two basic differences involved in the organization of the clinical studies with vaccines and drugs.

In drug trials, the subjects are often rejected due to the lack of trial efficacy for the particular patient. In vaccine trials, this concept is not used, the rejection of subjects is subject to other criteria (e.g. unexpected response to first or subsequent doses).

Another principal difference between vaccine and drug trials is the possibility of estimating or assuming the missing data before completion of a vaccine trial based on preliminary data, as opposed to drug trials where this is not possible. Moreover, a 
positive immune system response of the organism to the first dose of vaccine almost always results in a positive immune response to the subsequent dose (2). In order to include the particular vaccine into the immunization programme, a key issue from the viewpoint of public health is the decision-making process used to setup the national vaccination (dosing) schedule.

National immunization programmes in the world differ significantly not only with respect to their technical and financial organization. More importantly, there is a difference in the explanations and directives provided to the lay public and in vaccination schedules for vaccines of the same antigen composition. The same combination of antigens from different vaccine manufacturers are used in various schedules in the same countries.

In recent years, several new combined vaccines were included into the national immunization programmes, which are administered either independently or simultaneously. In 72 countries, the World Health Organization (WHO) registers a total of 29 different vaccination schedules which include only the basic diphtheria and tetanus vaccination. Despite the fact that different vaccination schedules are explained by different epidemiology of the diseases, funding, politics, distribution, etc., not all explanations are logical and understandable (3).

To determine the optimum age for initiation of vaccination against a particular disease, many factors are used that may influence this decision. These include age-specific incidence of the disease, risk of complications of the disease as well as the vaccination, capability of the organism to partially respond to immune stimulus, and possible interference with maternal antibodies or other antibodies acquired by artificial means (blood transfusion, blood product transfusion, etc.) (4).

Based on WHO recommendations, the primary vaccination against the basic diseases diphtheria, tetanus and pertussis in developed countries begins in the 6th week, followed by second dose in the 10th week, and 3rd in the 14th week of life. Decisions for this schedule were based on epidemiological situations in these countries, especially from the viewpoint of early vaccination against pertussis. Primary vaccination schedules in the rest of the countries (EU, USA, Canada, Australia, etc.) are based on two or three doses at various time intervals. Timing of the vaccination schedules is one of the most visible and most controversial professional problems of vaccination. Some countries (e.g. Italy, Scandinavian countries) give two basic vaccination doses (in 3rd-5th month) and the third dose is given no earlier than in the 11th month of age. However, the belief that the third administered dose can be also considered the first booster dose is in this case a purely academic issue (5).

Some countries, e.g., Austria $(3,5)$, France $(2,4)$ and Slovakia $(3,5)$, use identical or very similar schedules to give the first two doses and subsequently the third dose but only full administration of all three doses is considered a complete primary vaccination. The most controversial issue for the EU countries is also the question of early and sufficient protection against pertussis. The European Centre for Disease Prevention and Control (ECDC) recommends as the optimum model for EU countries an introduction of a uniform vaccination schedule - in 2nd, 4th and 11th month (5).

When comparing individual schedules, there is so far no relevant evidence that would definitively support any particular vaccination schedule. The problem is not just in the difference of the vaccination schedules but also a possible lack of confidence on the part of general public (6). Therefore, the monitoring of factors that may influence individual decisions in vaccination is as important as the monitoring of the remaining basic parameters of the immunization programme (population vaccination rate, efficacy of vaccination, safety, vaccine distribution, cost issues). Both factors can significantly influence the variations in vaccination schedules. The harmonization of the vaccination schedules within the EU should not be the main issue for the administrators of the vaccination programmes. The most important aspect is the comprehensible and acceptable explanation of the differences to general (and professional) public (5).

One essential condition when determining the immunization schedule is the safety of vaccination. Several studies confirmed that all implemented vaccination schedules for basic vaccination of children are safe and there is no difference in the frequency of vaccination adverse effects (7).

Another issue is the question of maximum age for administration of a particular vaccine. Internal properties of the vaccine are considered reactogenicity of the vaccine (higher content of the antigen for children, number of administered doses vs. reactogenicity) (8) as well as external conditions such as incidence and severity of the disease at older ages (e.g. Hib in children older than 5 years, etc.).

Detail specification of the European Medicines Agency (EMA) requirements for new antigens characterized by humoral type of immune response are in the Guideline on Clinical evaluation of new vaccines (9). One of the basic attributes of a successful immunization programme, in addition to available effective vaccine, is also decision on suitable vaccination schedule for a particular community (country). It should take into consideration not only the characteristics of vaccines or their combinations, age of the selected group, epidemiological situation of the diseases against which we plan to vaccinate, age-specific incidence, immune "status" of the population including maternal antibodies, and "herd" protection and other public health parameters $(10,11)$.

Vaccination schedules for basic vaccination are not rigid but they have certain flexibility; the basic vaccinations may be effectively and safely initiated from the age of six weeks. The latest ECDC recommendation for vaccination against diphtheria, tetanus, and pertussis, including a combination with vaccination against polio, Hib and HBV is the 2, 4, 12-month schedule (6). Vaccination before the age of six weeks is not recommended, with the exception of vaccination against tuberculosis (TB) and type $\mathrm{B}$ hepatitis (4th day or immediately after birth).

According to EMA, it is not necessary to study every possible schedule, but it is necessary to acquire relevant data for both basic schedules. For different applications of the three doses of the basic vaccination administered during the first 6 months of age, if sufficient immune response was found for a more challenging schedule (e.g. 2, 3 and 4 months or the Expanded Programme on Immunization (WHO EPI) schedule starting at 6 weeks of age), it is possible to extrapolate the data schedule with less condensed schedule (2, 4, 6 months; 3, 5 month sect.). On the other hand, it is not possible to extrapolate data acquired from less condensed schedules (2, 4, 6 months; 3, 5 months) for a "more challenging" schedule, starting at e.g. 2, 3, 4 or 6, 10, 14 weeks. Different schedules of the basic and booster vaccinations in the EU states result in a different number of total administered number of vac- 
cine doses containing DTP before the age of 18, between 4 and 7 administered doses $(10,12)$.

For basic vaccination from the viewpoint of efficacy and "cost-benefit," ECDC considers the administration of three doses at the age of 2, 4 and 10-11 months (4). Administration of two doses at the age of 2, 4 months results in a strong response with simultaneous administration of other vaccines and results in the assumption that other "more challenging" schedule (2, 3, 4 months; 2, 4, 6 months or 3, 5 months) will be sufficiently immunogenic. Similar principles are applied in the decisions on the EU vaccination schedule by EMA. According to EMA, the decision on a vaccination schedule should take into consideration the nature of antigen, target population (e.g. children, travelers, elderly), kinetic profile of the antigen response, and valid local national official schedule recommendations (9). If the vaccination schedule does not comply with for any reason, it is desirable to complete the basic vaccination as soon as possible. Primary vaccination against DTP, HBV, polio, and Hib in the EU and in the world are based on different immunization schedules; week 6, 10, 14 (WHO schedule), month 2, 3, 4 (Czech Republic), 3, 5, 11 (Sweden, Slovakia, Austria), and month 2, 3, 4 without booster (UK) (13).

The decision on the vaccine continuation or invalidity is based on the immunological response of the organism, most often on the basis of reduced titer of antibodies to minimum protective titers in time correlation. With respect to shortening of the recommended interval between two doses, the acceptable shift is within the range of $\leq 4$ days (grace interval). That means, when the dose is administered five days before the interval specified in the Summary of Product Characteristics (SPC), for example, this vaccination is considered invalid and must be repeated (14). In clinical practice, sometimes the interval between individual doses is shorter than recommended or the vaccination was started at the age lower than recommended. It is essential that the national healthcare authorities issue accurate instructions (guidelines) for such cases for the vaccinating staff.

In general, all regulating healthcare authorities accept each administered vaccine dose as valid and essentially they do not recommend initiation of a new vaccination series from the start provided that two essential requirements were complied with:

- Minimum age for antigen application, depending especially on its intrinsic characteristics (polysaccharide, protein, conjugated protein, recombinant DNA/RNA, live attenuated, etc.) and possible interference with maternal antibodies.

- Minimum delay for administration of a particular antigen.

From this viewpoint, a single SPC format could be defined for vaccines, establishing minimum age; minimum time period between individual doses ("most challenging schedule"); minimum number of vaccine doses in the basic vaccination series; and minimum delay for booster dose and related frequency (15).

In all other "less condensed" schedules national authorities should be responsible for immunization programmes.

According to the Mission Statement of the European Network of Paediatric Research, EMA will facilitate studies in order to increase availability of medical products authorized for use in the paediatric population (16).

Therefore, it is principally a question of ethics, considering that there are arguments supported by expert evidence for a possible extrapolation of the data on the immunogenicity of vaccines acquired in the trials with "most challenging schedules" to require additional trials on small children with the same or very similar vaccine in other "less condensed" schedules.

Along the same lines, differences in the SPCs of antigensimilar or identical vaccines may give rise to impression that in the case of one vaccine the application process "must" be rigorous and accurate. For example, the postponement or skipping of a particular dose results in invalid vaccination and, in the case of another vaccine, the administration of the dose is principally only "recommended" i.e. that the vaccination carried out before this moment is valid. Corrections for delays in the particular national immunization schedule are recommended by the national authorities - immunization committees - based on their own findings (immunology reviews), post-registration studies, etc. (9).

The vaccinating staff should adhere to the national vaccination schedule as much as possible. If the recommended schedule cannot be adhered to (e.g. due to temporary contraindication, failure to show up for vaccination visit, etc.), the medical staff should primarily ensure that all previous doses were administered after minimum recommended age and in line with the minimum intervals between doses.

From this point of view, there is a much higher risk of damage to a child resulting from delayed administration or failure to administer a vaccine. Community protection provides only limited protection to non-vaccinated individuals and, when it drops below estimated herd immunity threshold (e.g., 83-94\% measles, $92-94 \%$ pertussis, $80-86 \%$ polio, $75-86 \%$ mumps), this may lead to increased risk of transmission of an infectious agent within community. Its value varies with the virulence of the disease, the efficacy of the vaccine, and the contact parameter for the population (17).

Who determines the vaccination schedule? For the purposes of the authorization process using either centralized or decentralized procedure several dosing schedules are usually provided in SPC for vaccines. The authorization authority, e.g. for USA - the Centers for Disease Control and Prevention (CDC) sets the U.S. childhood immunization schedule based on recommendations from the Advisory Committee on Immunization Practices (ACIP). This schedule is also approved by the American Academy of Pediatrics (AAP) and the American Academy of Family Physicians (AAFP), it provides the national body competent to determine the national immunization programme the most optimal vaccination schedule for inclusion of a specific vaccine in the vaccination calendar of a specific country (18). In the EU countries, the standard authorities for organization of vaccination programmes and vaccination schedule are established by the public health authorities either at the health ministry or independently (19).

\section{CONCLUSIONS}

There is no official health recommendation as to when and how to start vaccinations before the recommended age in EU countries. According to published information, this is possible only in very specific cases if it is necessary to obtain the earliest possible protection of the child such as when travelling, for epidemiological reasons, or health reasons of the child. According to SPC, an important determining factor to use a vaccine before the specified age is the characteristic of the vaccine (inactivated, live, 
polysaccharide, conjugated, etc.), e.g. DTP vaccine - not before 6 weeks of age, MMR from 12 months but HBV on the day of birth.

On the other hand, there is no official recommendation for the latest age to start immunization in the EU. The primary series of immunizations against DTP in EU countries varied from six weeks to 12 weeks (2-3 months) of age. Variations for the application of the second dose are from four weeks to eight weeks and for the application of the third dose from 4 weeks to 6 months and more.

For the regular vaccination programmes, many different schedules are used in the EU. The number of DTP doses and combinations we need for primary series 2 or 3 has been discussed. We will accept a two-dose regiment for primary series and the third doses can be delivered 6 months at the earliest after the second doses. Every longer interval without any restrictions will be valid. The only question is whether two doses of the pertussis vaccine without booster during the second year of life will be sufficient to provide long lasting immunity.

According to SPC, for some DTP-combined vaccines, two doses are sufficient to ensure long lasting immunity but a minimum Hib booster during the second year is necessary. According to this schedule, only two doses of DPT (and combinations) at three and five months of age are satisfactory to induce long-term immunity for all other antigens (DTP, HB, and polio).

Compared to other drugs with accurate and exclusive dosage schedules, SPC for vaccines accepts more schedules (e.g. 2-5 different schedules for DTP) for application and additional schedules for "catch up" vaccination. Most of the vaccines from SPC contain the recommendation: "The use of this vaccine should be in accordance with official recommendations". Logically, according to this statement, it is possible to use a vaccine according to any official recommendation without official clinical studies.

Some general requests for "good vaccination practice" can be recommended:

- In a catch-up schedule, it is not beneficial to extend the interval more than recommended as the protective effect may be delayed, but if for any reason the vaccination series is interrupted, the vaccination should continue as soon as possible to complete the recommended schedule. It is not necessary to start the vaccination again from the beginning as the immunological memory ensures that the complex response to the subsequent doses is not reduced (even with longer intervals).

- Administration of vaccines at intervals shorter than minimum recommended intervals (not specified for the schedule but for a specific antigen), is not acceptable as it may result in reduced immune response. The minimum accepted interval for inactivated vaccines DTP and combinations between two doses is in general four weeks (28 days). The minimum valid interval (grace limit) for the administration of dose is max. four days earlier (inclusive), the administration of dose with interval shorter than five days and more $(28-4=24$ days $)$ is unacceptable and the vaccination (administered dose) is invalid, and vaccination (dose) must be repeated.

- Absolutely unacceptable is the administration of a vaccine at an earlier age than the minimum application age for the specific vaccine: e.g. DTP vaccine not before six weeks of age, MMR (measles, mumps and rubella) vaccine not before completed 12 months of age.

- In exceptional situations, like epidemics outbreaks or the case of post-exposition prophylaxis, it may be administered earlier (e.g. 6 months in USA), but appropriate dose must be administered at the age of at least 12 months.

- Three doses of vaccine are required for complete basic DTP vaccination, containing at least DT (pertussis is not administered only when contraindicated). In general, the antibody titer testing after vaccination is not required (e.g. when the recommended interval is extended). It may, however, be required in case of certain diagnoses (cancer, immune disorders, etc.).

Compared to drugs, vaccines cannot use pharmacokinetics or pharmacodynamics to define effectiveness of the vaccine, seroconversion, seroprotectivity and vaccine efficacy are the basis for the determination of the vaccination schedule for vaccines. ECDC and EMA accept great flexibility of vaccination schedules for vaccines for basic vaccination and for booster doses DTP and combinations vaccines. The only two necessary conditions essential for vaccine use in practice are the minimum age for effective dose administration of vaccine and minimum interval between vaccine doses. In general, authorities accept extrapolation of data determined from "more challenging" schedules for "less condensed" schedules but not the other way around (from "less condensed" to "more challenging") and practically "unlimited" maximum interval between doses (not only humoral response but also immune memory).

In the light of published information, the question is whether we need more than the "more challenging" schedule in SPC. In regard to SPC, more schedules include recommendations that a "vaccine should be used according to national recommendations," the local health authority (immunization committee) is only responsible to make final decisions for the national schedule, despite SPC recommended schedule. Optimalization of the vaccination schedule must be the exclusive responsibility of public health/ epidemiology, economics, political will, and vaccine availability. Finally, the ethical question is whether we need clinical studies with virtually identical composition vaccines for children for each schedule used in EU countries.

\section{Acknowledgements}

We would like to acknowledge Mary Talalay, MS MPH, (Johns Hopkins University) for the editorial assistance of this publication.

\section{Authorship Contributions}

Manuscript writing and literature research: VO, ZK and MŠ.

\section{Conflict of Interests}

None declared

\section{REFERENCES}

1. WHO Collaborating Centre for Drug Statistics Methodology. Guidelines for ATC classification and DDD assignment 2013. 16th ed. Oslo: Norwegian Institute of PublicHealth; 2012.

2. Mehrotra DV. Vaccine clinical trials - a statistical primer. J Biopharm Stat. 2006;16(4):403-14.

3. Larson HJ, Cooper LZ, Eskola J, Katz SL, Ratzan S. Addressing the vaccine confidence gap. Lancet. 2011 Aug 6;378(9790):526-35.

4. Kroger AT, Atkinson WL, Marcuse EK, Pickering LK, General immunization practices. In: Plotkin SA, Orenstein WA, Offit PA, editors. Vaccines. 6th ed. London: W.B. Saunders; 2013. p. 93-94.

5. Theeten H1, Nohynek H, Coenen TM; European Science Advisory Network for Health (EuSANH). EuSANH workshop "Reasons behind the differences in national vaccination schedules for under-five", European 
Public Health pre-conference workshop, Malta, 8 November 2012. Vaccine. 2013 Oct 1;31(42):4694-6.

6. European Centre for Disease Prevention and Control. Scientific panel on childhood immunisation schedule: Diphtheria-tetanus-pertussis (DTP) vaccination. Stockholm: ECDC; 2009.

7. The childhood immunization schedule and safety: Stakeholder concerns, scientific evidence, and future studies. Washington, D.C.: The National Academies Press; 2013.

8. Hambidge SJ, Newcomer SR, Narwaney KJ, Glanz JM, Daley MF, Xu $\mathrm{S}$, et al. Timely versus delayed early childhood vaccination and seizures. Pediatrics. 2014 Jun;133(6):e1492-9.

9. Guideline on Clinical evaluation of new vaccines. EMEA/CHMP/ VWP/164653/2005 [Internet]. London: EMEA; 2006 [cited 2014 May 22]. Available from: http://www.ema.europa.eu/docs/en_GB/document_library/Scientific_guideline/2009/09/WC500003870.pdf.

10. Lopalco PL, de Carvalho HG, Kreidl P, Leitmeyer K, Giesecke J. Childhood vaccination schedules in Europe vary widely Is this a problem? Bundesgesundheitsblatt Gesundheitsforschung Gesundheitsschutz. 2009 Nov;52(11):1095-8.

11. Kulldorff M. Study designs for the safety evaluation of different childhood immunization schedules. In: The childhood immunization schedule and safety: Stakeholder concerns, scientific evidence, and future studies. Washington, D.C.: The National Academies Press; 2013. p. 161-90.

12. D'Ancona F; Venice II Group. VENICE II: Go on combining our efforts towards a European common vaccination policy! Euro Surveill. 2009 Mar 26;14(12);pii:19161.

13. Chlíbek R, Smetana J, Boštíková V, Špliňo M, Hanovcová I. Vaccination schedule in Czech versus EU. Vakcinologie. 2013,7(3):108-14. (In Czech.)
14. National Center for Immunization and Respiratory Diseases. General recommendations on immunization - recommendations of the Advisory Committee on Immunization Practices (ACIP). MMWR Recomm Rep. 2011 Jan 28;60(2):1-64. Erratum in: MMWR Recomm Rep. 2011 Jul 29;60:993.

15. Wiese-Posselt M, Reiter S, Gilsdorf A, Krause G. Needs and obstacles of uniform immunisation schedules in the European Union. Bundesgesundheitsblatt Gesundheitsforschung Gesundheitsschutz. 2009 Nov;52(11):1099-104.

16. European Medicines Agency [Internet]. London: EMA; 2014 [cited 2014 Jun 5]. European Network of Paediatric Research at the European Medicines Agency (Enpr-EMA). Available from: http://www.ema.europa.eu/ ema/index.jsp?curl=pages/partners_and_networks/general/general_content_000303.jsp.

17. Fine PE. Herd immunity: history, theory, practice. Epidemiol Rev. 1993; 15(2):265-302

18. Centers for Disease Control and Prevention [Internet]. Atlanta: CDC; 2013 [cited 2014 May 22]. For parents: vaccines for your children. Available from: http://www.cdc.gov/vaccines/parents/sets-schedule.html.

19. TheVaccine European New Integrated Collaboration Effort (VENICE) III project [Internet]. Rome: Istituto Superiore di Sanità; 2014 [cited 2014 Oct 22]. Available from: http://venice.cineca.org/the_project.html.

Received November 10, 2014 Accepted in revised form January 11, 2015 